\title{
EINFÜHRUNG IN DIE THEORETISCHE PHYSIK
}

\author{
I \\ MECHAN I K
}

von

\author{
DR.-ING. WERNER DÖRING \\ o. Prof. an der Universität Hamburg
}

\section{Mit 23 Abbildungen}

Dritte, verbesserte Auflage

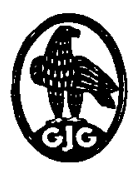

WALTER DE GRUYTER \& CO.

vormals G. J. Göschen'sche Verlagshandlung · J. Guttentag, Verlagsbuchhandlung - Georg Reimer - Karl J. Trübner · Veit \& Comp.

B E R L I N 1965 
Die Gesamtdarstellung umfaßt folgende Bände:

Band I: Mechanik (Band 76)

Band II: Das elektromagnetische Feld (Band 77)

Band III: Optik (Band 78)

Band IV: Thermodynamik (Band 374)

Band V: Statistische Mechanik (Band 1017)

Zur Schreibweise der Formeln:

Alle Formelbuchstaben dieses Buches bedeuten physikalische Größen, also Produkte aus Zahlenwert und Einheit, die von der Wahl der Einheit unabhängig sind.

Vektoren sind durch Fettdruck gekennzeichnet.

$\boldsymbol{a} \cdot \boldsymbol{b}$ bedeutet das skalare Produkt der Vektoren $\boldsymbol{a}$ und $\boldsymbol{b}$.

$\boldsymbol{a} \times \boldsymbol{b}$ bedeutet das Vektorprodukt der Vektoren $\boldsymbol{a}$ und $\boldsymbol{b}$.

Eine Ziffer in Klammern verweist auf eine Formel des gleichen Paragraphen. Andere Hinweise auf Formeln enthalten Paragraphund Formelnummer.

(C)

Copyright 1965 by Walter de Gruyter \& Co., Berlin 30. - Alle Rechte, eins chl. der Rechte der Herstellung von Photokopien und Mikrofilmen, von der Verlagshandlung vorbehalten. - Archiv-Nr. 7740650. - Satz und Druck: Walter de Gruyter \& Co., Berlin 30. - Printed in Germany. 